\title{
Arqueología e Historia del Curso Medio e Inferior del Río Aconcagua: desde los Primeros Alfareros hasta el Arribo de los Españoles (300 aC-1600 dC). Fernando Venegas Espinoza, Hernán Ávalos González y Andrea Saunier Saunier. Ediciones Universitarias de Valparaíso, Pontificia Universidad Católica de Valparaíso, 2011, 259 pp.
}

\section{Reseñado por Pamela Fernández ${ }^{1}$}
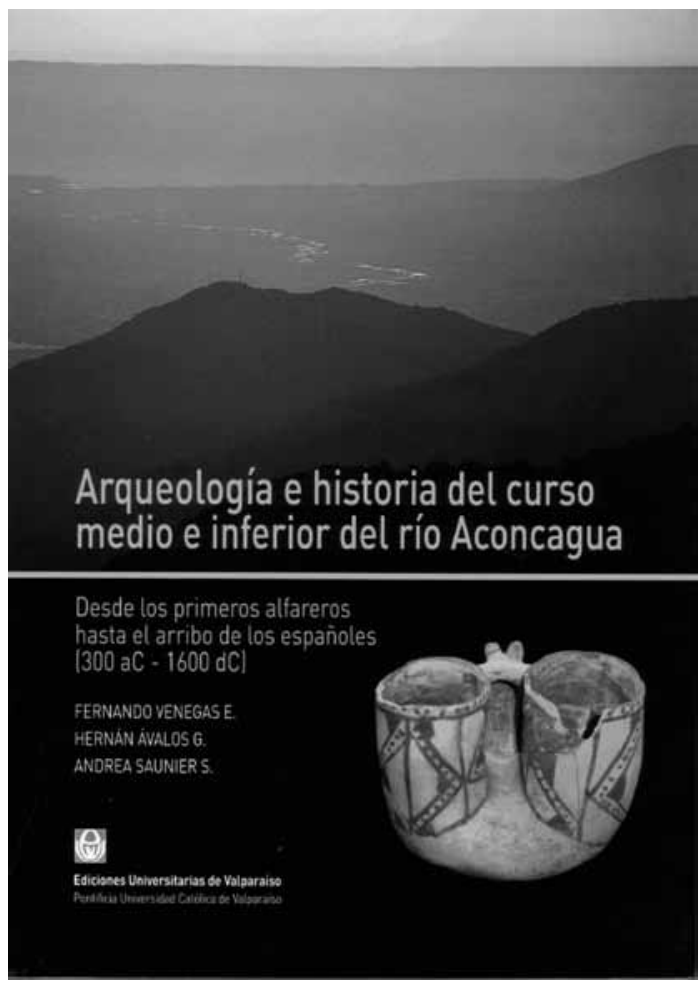

¿Prehistoria o Historia? Probablemente este sea uno de los cuestionamientos que ha predominado a la hora de realizar investigaciones sobre las culturas americanas, puntapié inicial de la obra que nos aprestamos a analizar. Si bien los autores se han volcado al estudio de las poblaciones indígenas del curso medio-inferior del río Aconcagua, no han dejado de inquietarse por la orientación en que esa tarea investigativa ha de cristalizar. Así, el libro materializa un trabajo interdisciplinario en donde vemos concertadas las labores de la arqueología de manos de Hernán Ávalos; la bioarqueología a cargo de Andrea Saunier; y la reflexión histórica de Fernando Venegas. En definitiva, se congregan las distintas líneas de trabajo de estos investigadores locales con el fin de plantear una propuesta conjunta.

A grandes líneas, esta obra presenta el estudio de los pueblos prehispánicos de ese tramo del valle del río Aconcagua a través de dos vertientes: el estudio arqueológico y el análisis histórico, sin perder de vista los puntos de encuentro que permiten configurar un panorama amplio. Más aún, la obra en su conjunto es una invitación a reevaluar las fronteras que limitan y diferencian ambas ramas del saber, redundando en una separación abrupta entre el período prehispánico y el hispánico. En este sentido, la primera proposición que se esboza es la idea de abandonar ese tipo de conceptualizaciones rígidas y abogar por la colaboración de la Historia en la comprensión de las poblaciones indígenas prehispánicas, ya que esa época puede contemplarse hoy rebosada de historicidad. Y viceversa, el apoyo de la arqueología en el conocimiento de la fase posterior podría ser clave para interpretar los acontecimientos históricos ulteriores. De todos modos, estas primeras aproximaciones no son del todo resueltas, pues la línea discursiva se encamina a plantear interrogantes, dejando el debate en una situación abierta.

Entrando en los contenidos del apartado de arqueología, encontramos una sucinta revisión de la bibliografía concerniente al tema, así como una descripción metodológica y localización del área. Posteriormente se dan a conocer los procesos de cambio físico del valle del Aconcagua a través de la presentación de sus antecedentes geológicos. Aquí se examinan los distintos fenómenos naturales que intervinieron el comportamiento de las poblaciones del sector, ahondando en los elementos que permiten una reconstrucción paleoclimática.

En el segundo capítulo, Ávalos y Saunier se abocan al estudio arqueológico, introducido mediante un análisis general respecto de la existencia, valor y conservación de los sitios arqueológicos presentes en el área, señalando que:

la riqueza arqueológica de la zona, su alto poblamiento documentado desde el Período Alfarero Temprano en adelante, su ubicación geográfica estratégica, que la configuran como una zona de frontera, de tránsito y contacto entre culturalidades del Norte Semiárido y de la Zona Central del país, hacen de ella un objeto de investigación fundamental para entender la articulación entre los grupos prehispánicos no sólo del curso superior e inferior de la cuenca, sino también de Chile Central y hacia sectores trasandinos (p. 44). 
Lo anterior corresponde no sólo a una justificación de la temática de estudio en términos de marcos espaciales, sino también una suerte de corolario luego de la investigación de 31 sitios arqueológicos localizados en las comunas de La Calera, La Cruz, Quillota, San Pedro y en el curso inferior del río Aconcagua. Espacios donde las indagaciones realizadas incluyeron una observación de enterratorios, piezas cerámicas y líticas, además de un estudio bioarqueológico que permitió a los autores deducir elementos en torno a la alimentación, presencia de enfermedades y características de la actividad física.

Presentados los antecedentes arqueológicos, los autores proceden, en un tercer capítulo, a plasmar sus conclusiones. Para Ávalos y Saunier el material arqueológico disponible no permite configurar un patrón de asentamiento inequívoco para las comunidades Bato y Llolleo, por lo que advierten que sus enunciados corresponden más bien a aproximaciones y no a esquemas rígidos. Señalan que las diferencias entre ambas culturas estarían dadas por las características del entorno inmediato, lo que no solo implicaría una distinción en el ámbito económico, sino que, además, aquello se vería reflejado en la esfera de lo social y lo simbólico. Existe pues, una línea argumentativa coherente, ya que el estudio se inicia a partir de observaciones paleoclimáticas que luego son consideradas elementales para entender la elección de un determinado ecosistema, e incluso para evaluar el desarrollo de particularidades identitarias. Ahora bien, hilando más fino también podríamos hablar de un determinismo geográfico-ambiental en donde el hábitat o medio natural es un factor clave en la plasmación de los modos de vida de una cultura.

No obstante, se revela un esfuerzo de síntesis e interpretación, en donde diversos vestigios de la cultura material y/o situaciones rituales específicas se convierten en indicios sugestivos de identificación cultural. En definitiva, ese tipo de elementos permitieron caracterizar y diferenciar a la Cultura Bato del Complejo Cultural Llolleo, transformándose en claves comprensivas que posibilitaron la configuración de un esquema básico. Ahora bien, uno de los planteamientos fundamentales que Ávalos y Saunier infieren de la evidencia arqueológica se relaciona con el surgimiento de la Cultura Aconcagua a partir de las alianzas entre las culturas Bato y Llolleo. En este sentido, sostienen: "la tesis de una continuidad biológica entre las poblaciones Bato-Aconcagua en la costa y Llolleo-Aconcagua en el interior, y un cambio cultural asociado a nuevas condiciones sociales" (p. 120) relacionadas con cambios ambientales en el medio, lo que en definitiva confirmaría el origen local del poblamiento prehispánico durante el período Alfarero en Chile Central (p. 123). Estas afirmaciones si bien se apoyan en la reunión de evidencia multidisciplinaria, aun constituyen acercamientos preliminares que no logran dar una respuesta acabada a todas las interrogantes, pero que, sin embargo, establecen un antecedente valioso para las investigaciones siguientes. Lo anterior, ya que el estudio aportó nuevas aristas a la problemática, como la neutralización de los elementos identitarios entre Bato y Llolleo, no obstante la persistencia de diferencias sutiles entre lo Aconcagua costero de tradición Bato y lo Aconcagua del interior de origen Llolleo (p. 124). Lo que sumado a la necesidad de integrar estos resultados a los estudios del curso medio y superior del Aconcagua, trazan amplias perspectivas de trabajo.

Al llegar al apartado de Historia nos encontramos inmediatamente con un conciso recuento de lo que han sido las interpretaciones historiográficas en torno al impacto de la conquista española, preludio de lo que serán las páginas siguientes, "una revisión del significado que tuvo el avance de los inkas y por sobre todo de los ibéricos, sobre las poblaciones locales del Aconcagua" (p. 136), claro que sin abandonar la expectativa de abordar la nueva configuración socioeconómica gestada por los conquistadores. Así se enfatiza en el carácter microhistórico del estudio, sin descartar sus acercamientos con la historia local, distinguiendo entre una perspectiva local y otra localista (p. 135).

En el cuarto capítulo se revisa la expedición de Almagro, enfatizando las consecuencias de tal contacto y afirmando que "lo que no es cuestionable es que el impacto de la venida de Almagro entre la población local fuese considerable.[...] La población debió verse disminuida de modo significativo, además de quedar fuertemente conmovida" (p. 143). A la luz de esos antecedentes, señala Venegas, no resulta difícil entender la resistencia que ejercieron las poblaciones locales a la empresa de Valdivia, situación que determinaría la materialización de su proyecto en el valle contiguo. Como segundo punto, el autor analiza las características societales y económicas de los Aconcagua y los Mapochoes, recalcando los puntos que permiten comprender su estructuración social en el ámbito de la jerarquización grupal, las actividades económicas, entre otros aspectos elementales para evaluar la resistencia al conquistador español y hacia los incas. El autor se permite cuestionar la real influencia del dominio incaico sobre la cultura local, apuntando que existen diversos indicios (sobre todo en la esfera de lo simbólico religioso) que posibilitan identificar expresiones culturales de arraigo histórico local (p. 157), lo que no implicaría negar el influjo que el contacto produjo en la cultura material.

Un tercer aspecto examinado por el autor se relaciona con las distintas vías que las poblaciones locales concibieron para enfrentarse al dominio español. Así, perfila una línea divisoria entre la actitud más ofensiva de los Promaucaes y los Aconcagua frente a una posición más consentidora de los Mapochoes. Finalmente, este capítulo termina con lo que el autor identifica como una de las prioridades de Valdivia en el proceso de conquista, el control de la frontera del Aconcagua como estrategia primera para asentar la posterior colonización. Tal objetivo, se vería concretado en 
la construcción de la Casa Fuerte de Quillota, edificación que sería crucial en el posterior declive de la resistencia indígena y en la consolidación de la ocupación hispana. No obstante, Venegas sostiene que la sociedad que se fue conformando a posteriori va a responder a las dinámicas prehispánicas, ya que si bien los españoles intentaron encauzar ese proceso a través de lonkos, estos no pudieron detentar la autoridad de que fueron investidos.

En el último capítulo Venegas realiza una descripción del reordenamiento económico del Aconcagua, esclareciendo la función y los parámetros bajo los cuales surge la estancia de Pedro de Valdivia en el Valle de Chile. Este lugar se habría convertido en el centro de operaciones de la economía del Aconcagua, por lo que desde allí se habría organizado la actividad agropecuaria y las faenas de los lavaderos. Sin embargo, la presión sobre los territorios de la zona central a partir del siglo XVII habría determinado no solo su posterior desmembración, sino también constantes ventas de dominios indígenas de la zona del Aconcagua, debido a que, en teoría, las mercedes de tierra no podían realizarse sobre los terrenos de las poblaciones locales. No obstante, si bien esta situación correspondería a una práctica para consolidar el traspaso de tierras a los europeos, Venegas destaca el reconocimiento que se hacía en el marco legal de las posesiones que correspondían a los indígenas y a la posibilidad, al menos, de que estos últimos pudiesen, eventualmente, recuperar las tierras que hubiesen sido usurpadas.

En la última sección de este capítulo se presenta una documentación de las ventas y posesiones de tierras realizadas por los españoles, confirmándose una progresiva transformación del espacio desde terrenos indígenas de uso intensivo y extensivo hacia la conformación de estancias de carácter privado, las que, no obstante, mantendrían ciertas prácticas de uso común. Asimismo, a través de esos documentos se intentó comprender el funcionamiento de las comunidades nativas posterior a la conquista y ponderar las divergencias entre la estructuración prehispánica y la hispánica. Aunque si bien existieron diferencias en torno a la organización de la comunidades, desde la perspectiva del autor, lo cierto es que debido al interés que el indígena provocaba como mano de obra para el español, las comunidades pudieron mantener algunos de sus aspectos culturales que sobrevivieron al paso del tiempo mediante el sincretismo cultural y religioso.

En síntesis, es posible advertir que las líneas argumentativas de este trabajo pasan por esclarecer el panorama arqueológico e histórico de las poblaciones indígenas del curso medio e inferior del Aconcagua, desambiguando los aspectos sociohistóricos que en los estudios generales han permanecido inadvertidos. En este sentido, el estudio arqueológico permitió aportar detalles en torno a la configuración de la cultura Aconcagua y diferenciar los elementos constitutivos de las poblaciones precedentes, Bato y Llolleo. Por su parte, el análisis histórico posibilitó entregar claves comprensivas en torno a la respuesta indígena a la dominación española en el valle del Aconcagua, así como en relación con los procesos que más tarde modificaron la organización y entorno de las comunidades. Ahora bien, su valor no reside solo en el hecho de cuestionar los presupuestos que han girado a través de generalizaciones sobre estos pueblos, indicando el camino para otros estudios locales, sino que además responde a un debate mucho más amplio respecto de los imperativos epistemológicos que subrayan la pertinencia de estudios interdisciplinarios. Asimismo, este libro es también un llamado de atención sobre el potencial arqueológico presente en la zona y la necesidad de su efectiva protección sin importar intereses políticos o económicos. 



\section{La Ruralidad Chilena Actual. Aproximaciones desde la Antropología editado por Roberto Hernández Aracena y Luis Pezo Orellana. Colibris, Santiago, 2010, pp. 388.}

\section{Comentado por Debbie Guerra Maldonado ${ }^{1}$}

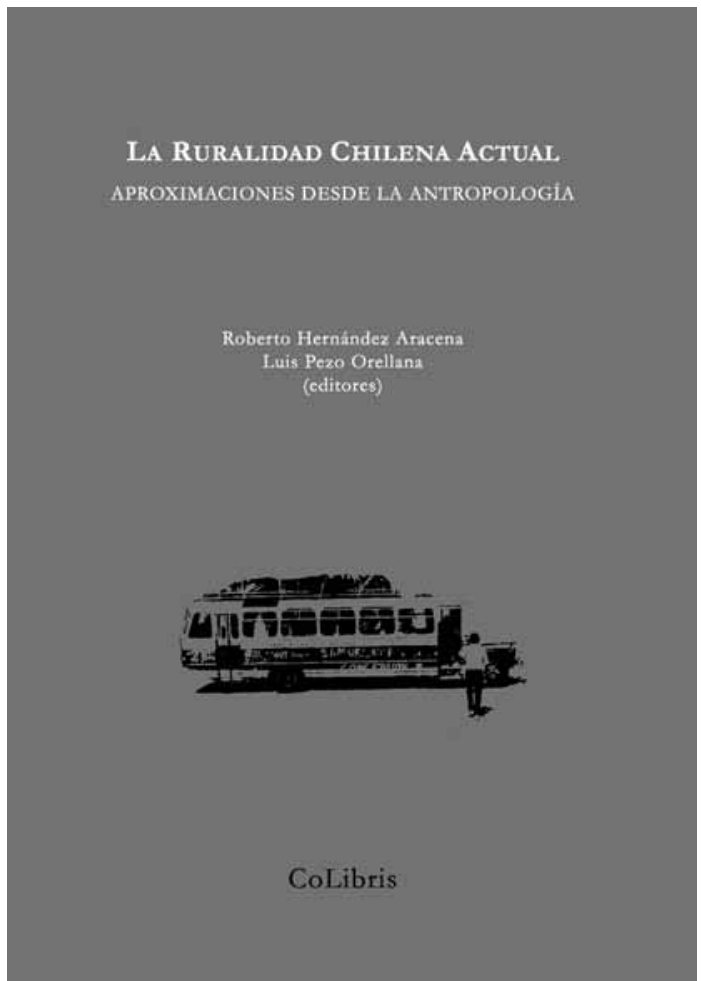

Lo que ocurre a la antropología rural es lo que, desde cierta perspectiva, suele pasar con la ruralidad: se homogeneiza y se invisibiliza. El sujeto rural desaparece. Esta desaparición no es inocente y representa opciones políticas declaradas, ante las cuales la antropología rural debiera pronunciarse. Para ilustrar este argumento voy a citar una opinión publicada en el Diario La Segunda del día 19 de octubre de 2010 sobre las políticas rurales, de Ronald Fischer, Profesor Titular de la Universidad de Chile, del Centro de Economía Aplicada del Departamento de Ingeniería Industrial, que, en parte, representa lo que pueden ser las orientaciones generales actuales del Estado chileno.

Fischer se pregunta: ¿Por qué debemos subsidiar a los pobladores rurales para que sigan viviendo en esos parajes? Su reflexión parte de la base según la cual la mayor parte de los/as habitantes rurales se dedican, desde su punto de vista, a una agricultura ineficiente, lo que les mantiene en condiciones de pobreza y de precariedad educacional. Los/as habitantes rurales, desde su perspectiva, son un peso para el sector público y su continuidad en los territorios es una prolongación de su pobreza.

¿Qué hacer?, se pregunta Fischer (2010). Plantea que las políticas rurales debieran estimular el éxodo hacia las ciudades, donde finalmente los empobrecidos habitantes rurales puedan encontrar la esquiva modernidad. Es en la ciudad donde podrán acceder a niveles de vida y educación aceptables. Lo que en consecuencia se requiere es transformar los subsidios rurales en subsidios a la movilidad. El argumento de Fischer (2010) importa consecuencias políticas no menores como, por ejemplo, que las políticas de tierras en el mundo indígena sean consideradas como erróneas: "Lo que se necesita es facilitar la transición a las ciudades y no entregar tierras que los mantengan en la pobreza porque son bienes que no se pueden vender" (Fischer 2010:21).

El libro La Ruralidad Chilena Actual. Aproximaciones desde la Antropología, una colección de artículos editados por Hernández y Pezo (2010) invita a matizar los planteamientos de Fischer, los que, en resumen, representan, por una parte, una afirmación tácita de una cierta forma de concebir lo rural, y, por la otra, una ceguera absoluta acerca de las especificidades de la ruralidad en nuestro país. El libro convoca, en este sentido, a ver lo que la política propuesta prefiere no ver: el mundo rural en su especificidad histórica, en su diversidad y en su posible protagonismo. La otra mirada, que es la mirada del libro, prefiero presentarla en relación con tres ejes de reflexión que surgen de la lectura de los trece artículos y la presentación que contienen las 388 páginas de la obra. Estos ejes son los de la subordinación de lo rural, su pluralidad, y la definición de su significado.

\section{Subordinación Excluyente y el Verticalismo en la Relación con lo Urbano}

Autores como Hernán Salas y Juan Carlos Rodríguez (en Hernández y Pezo 2010:45-78) invitan a revisar estas decisiones de orientación racional de máximo resultado y de máxima eficacia que están implicadas en el artículo de Fischer (2010). Los autores sugieren que existe una expansión del nuevo dominio de la acción racional, donde el espacio se plantea como un campo de acción instrumental específico, articulando de modo significativo el planeamiento de la ocupación humana. Una mirada

$1 \quad$ Instituto Salud Sexual y Reproductiva y Centro de Estudios Ambientales y Desarrollo Humano Sostenible. Universidad Austral de Chile, Valdivia, Chile. guerradebbie@ gmail.com 
como esta prescinde de la consideración del territorio como socialmente constituido y con ello renuncian a dos aspectos que son centrales: el territorio como espacio social apropiado y como espacio valorado.

El espacio es donde se desarrolla la identidad y se ejerce la pertenencia de los sujetos, dentro de un contexto que diferencia las formas de apropiación y valorización de cada grupo, de acuerdo con lo que el grupo y su cultura son: el territorio se define dentro de las estrategias patrimoniales de éste (Salas y Rodríguez en Hernández y Pezo 2010:69).

La lectura invita a recuperar no sólo la particularidad de las distintas escenas rurales, sino que también a aventurarse en el protagonismo siempre postergado de sus sujetos y a embarcarse en un imaginario que les permita permear las relaciones con el Estado y con el medio extralocal.

\section{Pluralidad de los Mundos Rurales, Identidad y Sujeto}

En la mirada oficial prima el esencialismo inspirado por el congelamiento metonímico a través del que se hace desaparecer la presencia del sujeto (Clifford 1999 [1997]). En la lectura oficial se congela e invisibiliza, por ejemplo, la importancia de la participación económica de las mujeres, acentuándose el sesgo patriarcal de la sociedad chilena. Se ignora, en ese enfoque, el control de las mujeres sobre los procesos de producción y participación, plantean Andrea Chamorro, Juan Pablo Donoso y Rafael Contreras (en Hernández y Pezo 2010), a lo que se podría agregar su contribución a la conservación de la biodiversidad por la vía del manejo de las semillas. En Río Hurtado, sugieren la autora y los autores recién citados, las instituciones públicas no reconocen a las mujeres como sujetos de proyectos productivos, de modo que el modelo de desarrollo impulsado desde el Estado las excluye, negando su participación efectiva en la economía local.

La contribución de Sergio González (en Hernández y Pezo 2010:111-134) abre un espacio importante para el ejercicio de la ciudadanía desde lo rural, rescatando de paso la posibilidad de entender lo rural como un estilo de vida abierto donde no se renuncia a la superposición de los contenidos urbano-rurales y que, por lo tanto, no está definido por lo puramente negativo o residual. En la compleja relación entre la identidad y la ciudadanía, leída en un artículo del autor, se abre la posibilidad de, al modo que lo plantea Anzaldúa (1999), vivir en la frontera (borderlands).

Este espacio de protagonismo y ciudadanía permite a Yanko González (en Pezo y Hernández 2010:201-232) y a Luis Pezo Orellana (en Pezo y Hernández 2010:179200) romper con el adultocentrismo de la antropología y del proyecto nacional urbano al considerar a las y los jóvenes rurales como sujetos, analizando sus dislocaciones y resignificaciones, con metodologías participativas. A su vez, Andrés Donoso (en Hernández y Pezo 2010:277-290) fija su atención en el papel efectivo de las organizaciones en los procesos de toma de decisión y advierte el debilitamiento de la sociedad civil. La riqueza de cada organización no se logra verter en perspectivas propias que las inviten como proponedoras y, por qué no, como implementadoras de políticas de desarrollo ajustadas a su dinámica y especificidad.

Sin embargo, la obra en comento plantea precaución respecto de las formas en cómo se canaliza la participación de los sectores rurales en las políticas públicas. La paradoja es que la participación implica su propia negación. Así se desprende de la lectura del texto de Marcelo González (en Hernández y Pezo 2010: 253-276), cuando se necesitan representantes, pero no se admite la representación. Es la necesidad de representantes que plantea la legislación chilena lo que condiciona, en el mundo indígena, la generación de tales representaciones, con lo que, junto con tensionar las relaciones locales, se impide permear desde lo rural-indígena la política pública. Se trata de políticas que buscan crear dirigentes para administrar el imaginario público.

\section{Los Significados de la Ruralidad}

Para Fischer (2011) la ruralidad, en lo social, tiene una connotación puramente negativa, y en lo económico, tiene un sentido puramente extractivo. Pero ¿qué se entiende por ruralidad? ¿Qué se quiere con ella y sus habitantes? Gonzalo Díaz Crovetto (en Hernández y Pezo 2010:79-110) invita a historizar los conceptos generados acerca de la ruralidad y con ello concretar o especificar los procesos en su complejidad. ¿Qué es lo que vale del medio rural? ¿El suelo explotable, el material extraíble, las especies depredables? ¿O bien es el paisaje, el valor ecológico, el sentido identitario o patrimonial? Lo rural, así visto, no es puramente residual y la especificación de la condición rural resulta urgente frente a la pura negatividad y subordinación con que, desde la postura oficial, se lo planea con su consiguiente destrucción.

Roberto Hernández y Carlos Thomas (en Hernández y Pezo 2010:135-178) plantean un modelo ecológico cultural para la educación rural, que permita avanzar en su especificación, integrando la dimensión ambiental para fomentar fórmulas cooperativas que rescaten valores comunitarios como la solidaridad y la cooperación y fortalezcan los sistemas sociales locales, protegiendo además los bienes patrimoniales. La educación rural implica recuperar la complejidad local para ponerla al servicio de estos propósitos.

En suma, podría concluirse, de acuerdo con lo planteado por Vanessa Rojas, que en términos de sus significados lo rural sigue siendo configurado a partir de los ojos citadinos, provocando una relación unilateral entre el campo y la ciudad, primando ante todo la construcción de significados desde la perspectiva urbana (en Hernández y Pezo 2010:235). 
Ello se traduce en lo que, desde la perspectiva de Hernández y Pezo (2010:16), se describe como el proceso de conformación de la llamada nueva ruralidad "signada por la imposición de un modelo de desarrollo hacia fuera, a pesar de las resistencias de varios sectores excluidos de este proceso". Esta imposición queda de manifiesto en la situación actual de la economía caprina tradicional, cuya obstinada insistencia en formas tradicionales de producción la ha convertido en objeto de una intervención pública -consistente con la lectura oficial propuesta por Fischer (2011) - orientada más a su extinción que a su desarrollo, como se desprende de la lectura de Stüdemanm (en Hernández y Pezo 2010:325-366).

Lo rural, en contraposición a la versión oficial que inspira a la política pública, reclama ser visto en su complejidad e intersección con el mundo urbano y global. Se trata de soluciones heterogéneas, generadas por actores diversos y diversas, que dan cuenta de modos de habitar el territorio a través de los que se preserva una buena parte de la naturaleza y los patrimonios. Estos modos reclaman visibilidad no sólo para su mera reproducción, sino para hacerse presente mediante una representación efectiva en los procesos de toma de decisión que les afectan directamente, como se sugiere en este libro.

Esta constatación impone el desafío al quehacer de la antropología rural de "reducir la fragmentación y dispersión del quehacer académico y profesional", a fin de realizar aportes significativos ... que favorezcan concretamente a las diversas poblaciones rurales" (Hernández y Pezo 2010:36-37).

Terminaré señalando que este libro marca varios hitos importantes para la antropología chilena: es el primer libro patrocinado por el Colegio de Antropólogos de Chile A.G. $\mathrm{Su}$ factura cuidadosa y extensa demuestra la dedicación y entrega de sus editores, Roberto Hernández Aracena y Luis Pezo Orellana. El libro representa, además, uno de los pocos compendios de antropología chilena cuya prolijidad y exhaustividad lo convierten en un referente obligado para estudiantes, investigadores/as, investigados/as y la comunidad en general. El volumen de la producción compilada por el libro habla de la vigencia de una reflexión importante, que permite sugerir que la antropología rural, lejos de de ser un tema agotado, es, como señalaba el lema del VII Congreso Chileno de Antropología (2010), una apertura a nuevos desafíos en la disciplina.

Las tareas planteadas por la propia obra a través de las reflexiones de sus editores invitan a identificar elementos diferenciadores del mundo rural, a fortalecer el desarrollo teórico en sus procesos de cambio y avanzar en los niveles de consolidación disciplinaria. Algunas preguntas siguen rondando: los temas de la exclusión, las relaciones de género e intergeneracionales, las migraciones estacionales y la naturaleza de la interfaz urbano-rural (incluyendo el impacto de lo global, el turismo y los nuevos estilos de unidades asociadas a la producción orgánica), son materias que convocarán por mucho tiempo a este destacado grupo de investigadores e investigadoras que representan prácticamente a todas las generaciones de la antropología chilena.

Celebramos el nacimiento de este libro no sólo porque nos reúne como comunidad antropológica, sino también porque es un testimonio profundo de la existencia de un sector de la sociedad que Chile pareciera preferir ignorar.

\section{Referencias Citadas}

Anzaldúa, G. 1999. Borderlands/La Frontera. The New Mestiza. Aunt Lute Books, San Francisco.

Clifford, J. 1999 [1997]. Itinerarios Transculturales. Traducido por Mireya Reilly de Fayard. Gedisa, Barcelona.
Fischer, R. 2010. Opinión: Políticas Rurales. Diario La Segunda (19 octubre 2010: 21).

Hernández, R. y L. Pezo (eds.) 2010. La Ruralidad Chilena Actual. Aproximaciones desde la Antropología. Colibrís, Santiago. 



\section{Dataciones radiocarbónicas NO deberían demorar una eternidad}

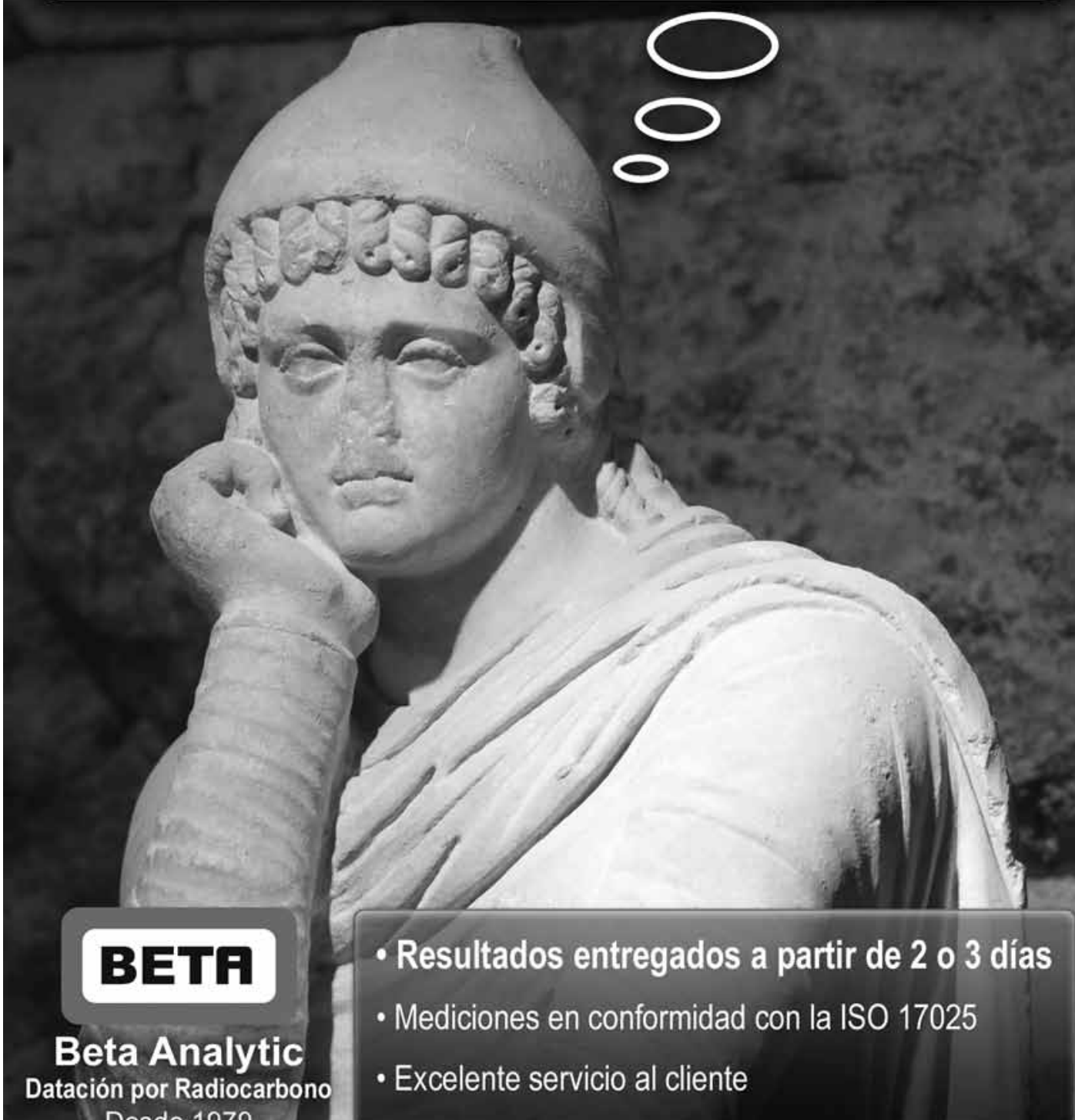

Desde 1979

\section{Australia Brasil China India Japón UK EE.UU} Visite www.radiocarbon.com para obtener detalles 

ANDROS IMPRESORES

www.androsimpresores.cl 
